\title{
PREDICTION OF ENERGY CONSUMPTION IN THE INDUSTRY 4.0 PLATFORM - SOLUTIONS OVERVIEW
}

\author{
Sebastian Temich, Artur Pollak, Jacek Kucharczyk, Wojciech Ptasiński \\ APA Group, Gliwice, Poland \\ e-mail: sebastian.temich@apagroup.pl (corresponding author); artur.pollak@apagroup.pl; \\ jacek.kucharczyk@apagroup.pl; wojciech.ptasinski@apagroup.pl \\ Arkadiusz MężYK, Damian Gąsiorek \\ Silesian University of Technology, Faculty of Mechanical Engineering, Gliwice, Poland \\ e-mail: arkadiusz.mezyk@polsl.pl; damian.gasiorek@polsl.pl
}

For a long time, scientific and technical work has been focused on production management, which affects both the correctness of the process and the costs generated. One of the integral elements of the production process management is energy, which has an impact on the organization of work, operation of machines or production. Predicting the energy consumption of smart facilities is crucial for implementing energy-efficient management systems, the area of this problem is a key aspect of smart grids whereby loads must be planned in real time. One of the main tasks of intelligent systems is to optimize the energy demand and costs to maximize energy efficiency of the facility. According to forecasting requirements, the following article presents several approaches to prediction of energy consumption models for production engineering systems. The proposed models were adopted and analyzed in terms of their usability and were trained and validated with the use of real data collected from the electrical installation of some company using the APA IPOE system.

Keywords: Industry 4.0, artificial intelligence, IPOE, production management, mechanical engineering, production engineering

\section{Introduction}

Nowadays, economy and automation of production systems depend on the available information technologies. Today, Industry 4.0 (I4.0) is not only a development slogan but is an industrial and academic activity. Countless companies and research organizations have begun working on this area with various aspects. Over the years, a standard has been developed in which the I4.0 system requires interoperability between all areas in the company and management awareness of the idea of running it (Bonilla et al., 2018; Wang et al., 2020). To achieve full integration, data analyzed from each process is necessary, being a medium between various production facilities and activities (Tkachenko et al., 2019; Pollak et al., 2020). In an I4.0 factory, data is gathered and tabulated to search valuable information, which can be captured and used to improve processes. Although production of an I4.0 factory can not only be integrated, automated, predictable and intelligent but also must be sustainable and renewable (Adenuga et al., 2020; Podder et al., 2020).

Analysis of mechanical and electrical signals is one of the most important areas of sustainable production development.

Global energy demand can be met by saving electricity, which is important for production planning as well as for improving awareness of electricity costs. Reducing energy consumption is one of the necessary research goals for sustainable production in the I4.0 era. Technological 
innovations affect energy consumption and management decisions regarding competitiveness of productivity. Due to growing demand for methods of analyzing energy consumption, the article attempts to solve the problems related to prediction of energy, which is an integral part of the development of a modern enterprise and is one of the most important aspects of the analysis of production signals (Gruber, 2013; Leme et al., 2020).

In a modern facility, data analysis can be performed using Intelligent Energy Consumption Platform (IPOE) created by APA Group (APAGROUP-1, 2021). IPOE allows one to measure parameters of the building energy system (i.e. total power) since which the current demand and consumption can be analyzed to increase the efficiency of production engineering.

Power prediction in each time window is aimed at detecting upcoming exceedances or anomalies that result in financial penalties imposed by the energy supplier or detection of upcoming changes. Correct prediction can prevent damage to machines or their temporary shutdown, which has a direct impact on the continuity of production. The algorithms presented in the following Sections are designed to predict the behavior of the tested signal in the near future. The design of the algorithms enables analysis of any type of the signal, mechanical or electrical (Ptasinski et al., 2021). In the presented publication, the accuracy of the proposed methods was analyzed for the electrical signal with the use of the IPOE platform. While using the discussed methods for mechanical signals, it is recommended to incorporate the NAZCA4.0 system (APAGROUP-2, 2021).

\section{Concise data analysis}

To ensure the quality of a production process, it is necessary to monitor the quality of electricity supplying individual machines on the production line (Bonilla et al., 2018; Gościniak and Wodarski, 2019). An adequately early response to the predicted energy consumption is an especially important issue in production engineering due to the maintenance of continuous operation of devices. As mentioned earlier, in the presented publication, the accuracy of the proposed methods was analyzed for the electrical signal with the use of the IPOE platform, while the NAZCA4.0 system is recommended when using the discussed methods for mechanical signals. The predictive approach involves measuring the power energy by a metering device located next to the main energy meter (Fig. 1). The measurement data is continuously processed by appropriate algorithms to carry out the energy consumption prediction process. Information about the estimated consumption in 15 minutes is sent to the management panel for further interpretation of the production process.

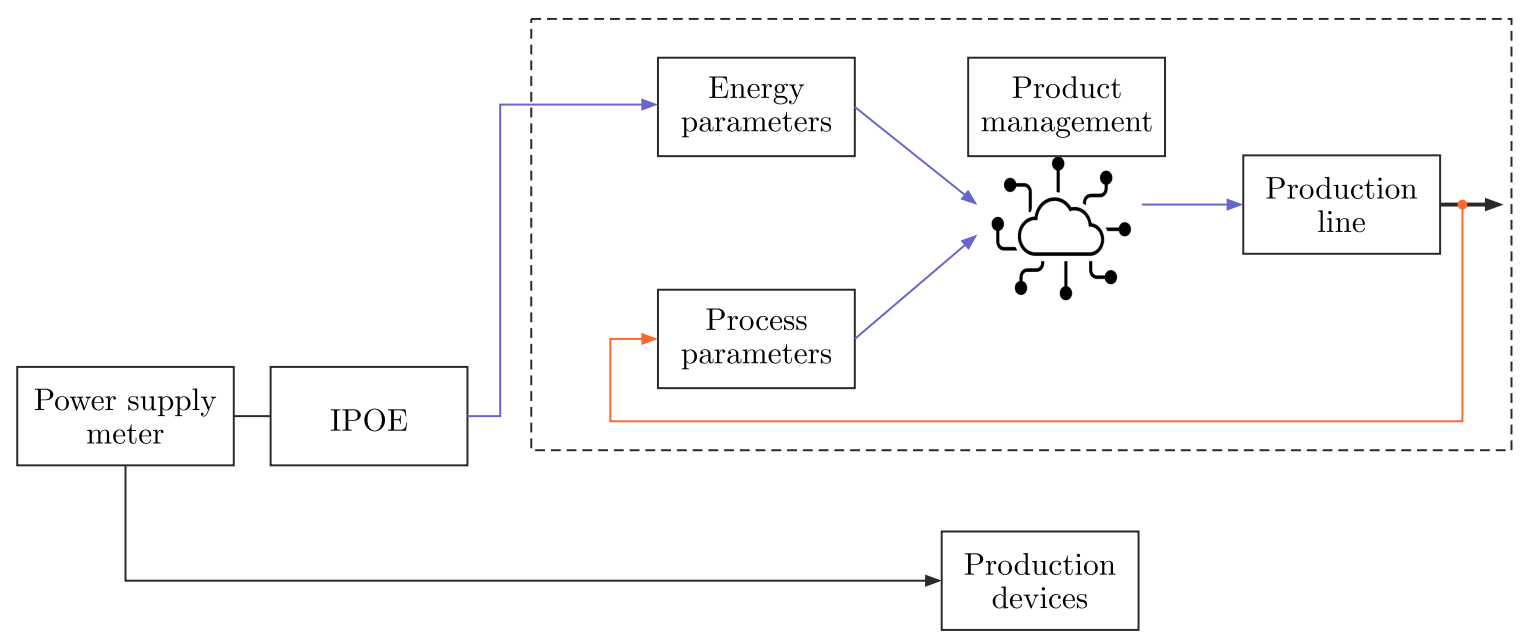

Fig. 1. Idea of the prediction system 
The prediction of active power consumption was carried out for one of the factories, where the IPOE platform aggregates data in the production facility. The platform allows for the analysis of data in real time, however, for the purposes of prediction, data averaged over a minute cycle was used, and the analysis was based on historical data from 05.11.2020-16.11.2020.

Figure 2 presents changes in the total active power over time. It could be stated that the power consumption in the analyzed facility is cyclical, the production takes place on working days, i.e., from Monday to Friday, which shows an increase in energy consumption, and ceases on non-working days, i.e., Saturday (e.g., 2020-11-07) and Sunday (e.g., 2020-11-15), for which the total active power does not exceed $100 \mathrm{~kW}$.

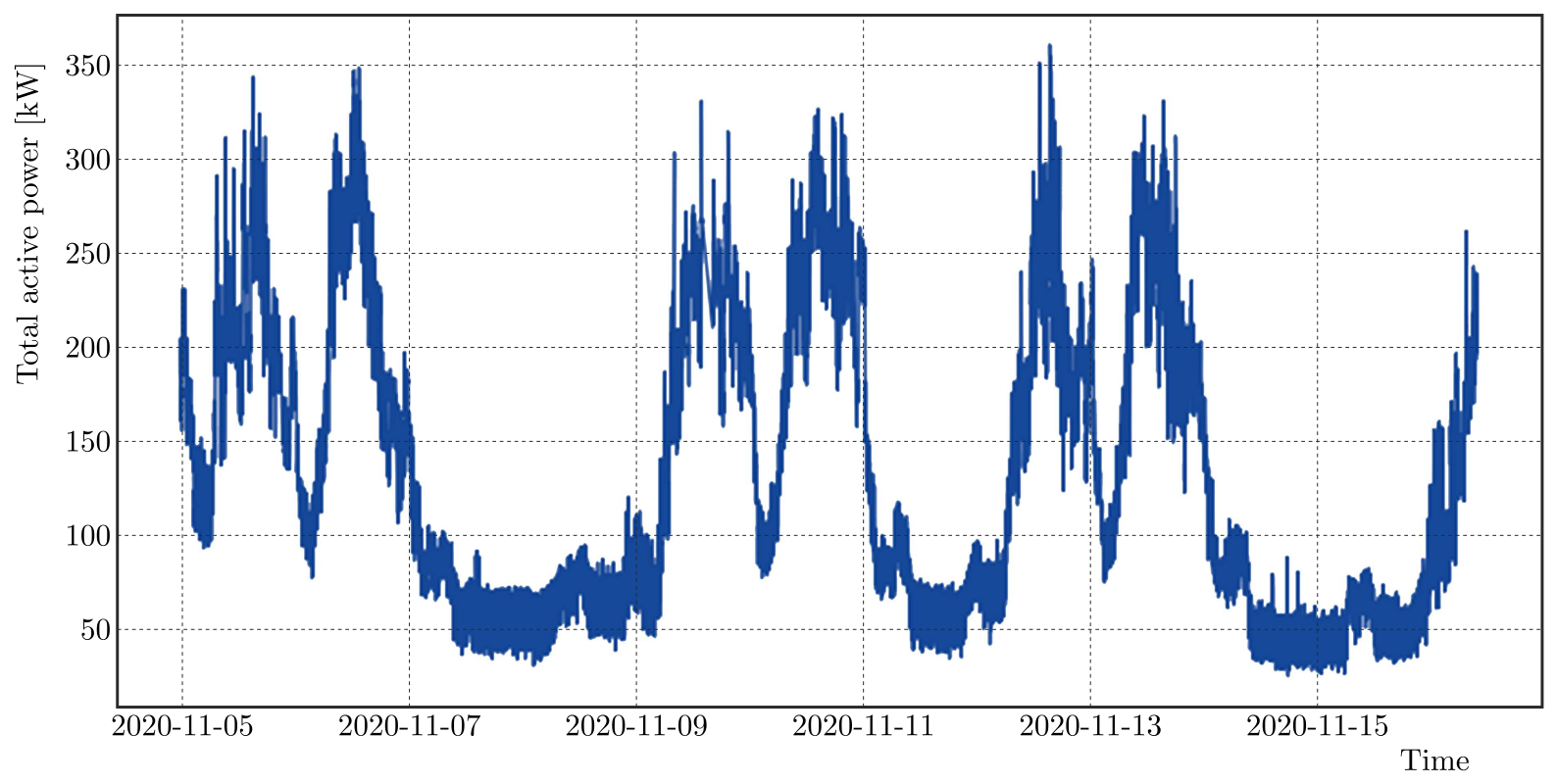

Fig. 2. Total active power in the assumed period of time

(a)

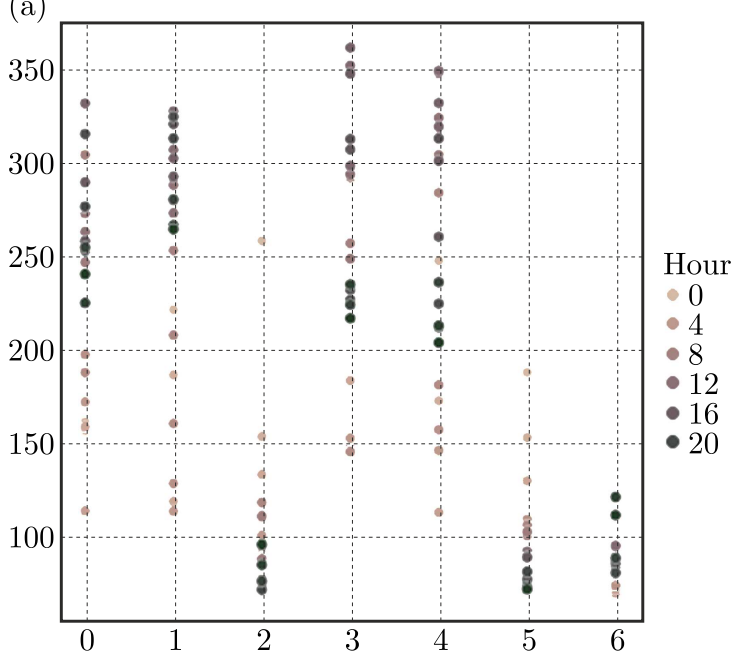

(b)

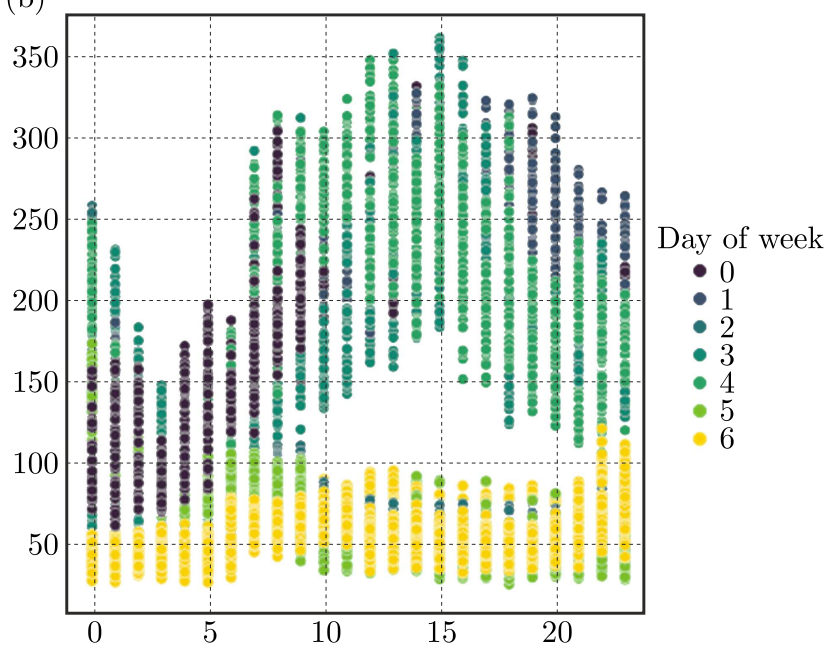

Fig. 3. (a) Active power in terms of hours for each day of the week; (b) maximum active power in weekly terms for each hour of the day

Figure 3a shows the maximum value of the total active power depending on the day of the week for each hour, and Fig. 3b depending on the hour for each day of the week. The increase in energy demand can be seen on 0 - Monday, 1 - Tuesday, 2 - Wednesday, 3 - Thursday. A decrease in the maximum active power on 5 - Saturday and 6th Sunday confirms the correctness 
of the application for production on working days. The deviation from the expected values on Tuesday 2nd (low value of the maximum power), may be due to a production break (e.g., a maintenance break), and 11th of November is a free of work day in Poland. The greatest demand for active power occurs from approx. 12 o'clock each day, suggesting that the plant is maximizing its production during these hours.

In the next step, a statistical analysis of the total active power in a selected time window was performed. Figure 4 shows the number of appearances of individual values of the active power. It should be concluded that the enterprise under normal operating conditions consumes no more than $100 \mathrm{~kW}$ of the active power. The low frequency of occurrence of the maximum power values leads to the conclusion that some of the devices are turned on only in certain cases or during the start-up process. The device requires a significant amount of power, and in the work cycle the power demand is a dominant component (e.g., the process of starting the furnace and operation in sustain).

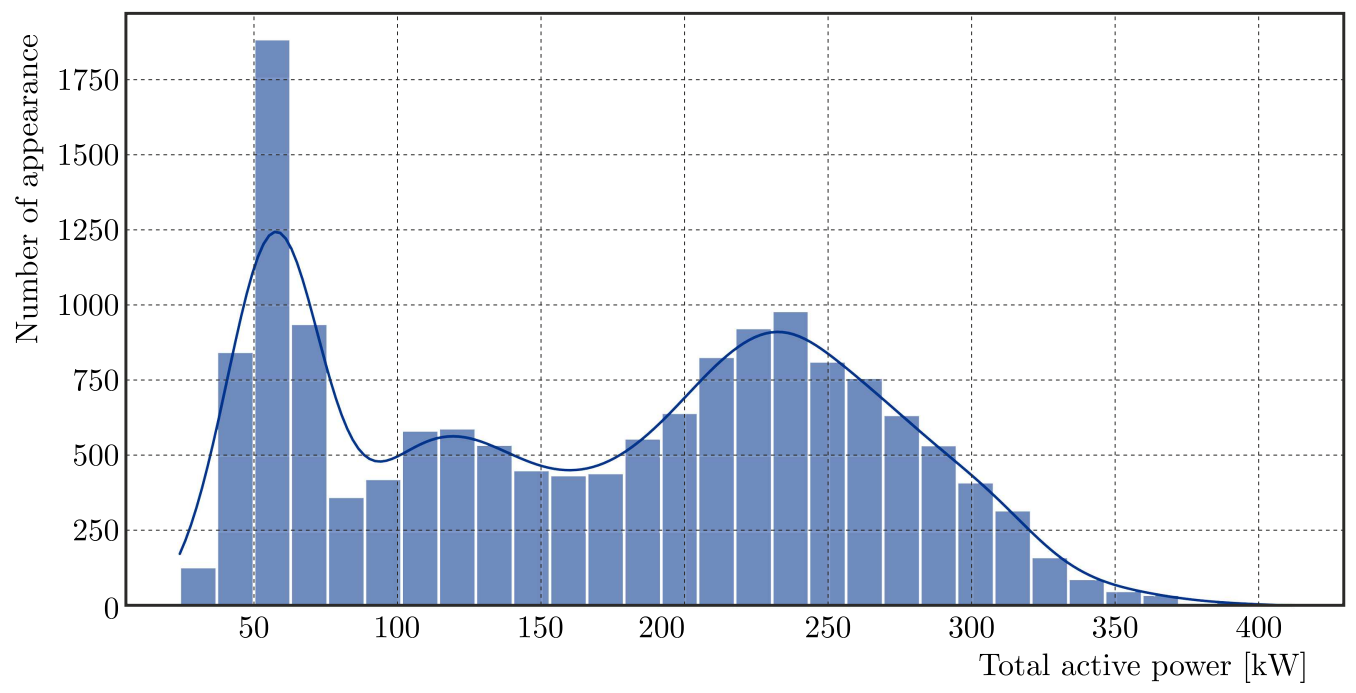

Fig. 4. Total active power histogram

Based on the statistical parameters (Table 1), it can be concluded that the average power (median) is approx. $103 \mathrm{~kW}$, the lower power quartile (Q1) is $64 \mathrm{~kW}$ and the upper one (Q3) is $194 \mathrm{~kW}$. The negative value of the curtain informs about data dispersion around the average, i.e., lack of concentration of the results, which is also confirmed in Fig. 4.

Table 1. Statistical analysis of the active power value in the assumed time interval

\begin{tabular}{|c|c|c|c|}
\hline Min power & $24.849 \mathrm{~kW}$ & Std & 76.641 \\
\hline Max power & $360.039 \mathrm{~kW}$ & Median & $103.167 \mathrm{~kW}$ \\
\hline Q1 & $64.894 \mathrm{~kW}$ & Kurtosis & -0.8703304786 \\
\hline Q3 & $194.12 \mathrm{~kW}$ & Mean & $131.703 \mathrm{~kW}$ \\
\hline
\end{tabular}

The conducted analysis allowed for the conclusion that the active power consumption in the enterprise is seasonal in nature, which enables introduction of regression methods to predict the total value of active power in the enterprise.

\subsection{Preparation of data for the prediction case}

The idea of predicting energy consumption is based on the use of historical data averaged over a minute cycle, available in a given time window, see Fig. 2. To verify the prediction methods, 4 sets of training data were prepared. Each set create a feature vector with historical values as 
predictors and $P_{t+15}$ as the predicted value. The first set contains only historical data prepared in accordance with Fig. 5 (historic data of the active power $P_{t}$ values of the last 5 minutes), the second set is repetition of the first one, but its values were normalized in the range 0-1.

\begin{tabular}{|l|l|l|l|l|l|l|}
\hline$P_{t-5}$ & $P_{t-4}$ & $P_{t-3}$ & $P_{t-2}$ & $P_{t-1}$ & $\longrightarrow$ & $P_{t+15}$ \\
\hline
\end{tabular}

Fig. 5. Data preparation idea

Based on the time analysis of active power waveforms, it was concluded that the information about the minute, hour and day of the week of a given value may be important in the forecasting process, so the next two training sets contain the indicated time information for each of the features. According to the second set of data, time info values were also normalized in range of $0-1$.

\section{Regression methods}

The main task of regression methods is to predict the energy in 15 minutes $\left(P_{t+15}\right)$ based on the features vector. Prediction may be possible because the energy consumption is cyclical, and with adoption to the historical energy consumption, the proposed algorithm will be able to determine a near future consumption. As a part of the analysis, 9 regression methods have been proposed and some of them will be discussed in this paper (the results of the rest will be presented in the summary of this paper). The following parameters were determined for each of the proposed models: analysis time, $R^{2}$ parameter, mean square error (MSE) and mean absolute error (MAE), which together constitute the model evaluation parameters.

\subsection{Facebook prophet}

Facebook prophet is a time series data forecasting tool based on an additive model in which nonlinear trends are adjusted to annual, weekly and daily seasonality and holiday effects. It works best with time series that have strong seasonal effects and several seasons of historical data. Prophet is immune to lack of data and trend changes. Prophet uses a decomposable time series model with three main components of the model: trend, seasonality and holidays (Díaz Redondo et al., 2020; Prophet, 2021)

$$
y(t)=g(t)+s(t)+h(t)+e(t)
$$

where $g(t)$ is the trend, $s(t)$ is the annual seasonality, $h(t)$ is the weekly seasonality and $e(t)$ is noise. As in the generalized additive model (GAM), several linear and nonlinear time functions are fitted as components. Forecasting the course of time is done in the form of a "curve-fitting exercise", not by timing each observation. The advantage of this approach is the ability to generalize the tool in the case of no or incomplete access to data. The prediction is performed using two test sets containing real and normalized data. The analyzed set of total active power values has been divided into subsets, i.e., training with 14000 measurements and a test with approx. 2000 measurements. Examples of values in the test and training sets are presented in Table 2.

The division of data into a training set and a test set was also presented graphically in Fig. 6, where the red color represents the training set and the blue color shows the power values in the test set.

According to the fact that the load in the plant changes in a cyclical manner, the weekly seasonality parameter was added to the prediction model. The model is extrapolated to the 
Table 2. Division of data into training and testing sets

\begin{tabular}{|c|c|c|c|}
\hline \multicolumn{2}{|c|}{ Training data } & \multicolumn{2}{c|}{ Testing data } \\
\hline Time & Power value & Time & Power value \\
\hline \hline 2020-11-05 00:00:00 & 203.958000 & $2020-11-15$ 00:01:00 & 32.714750 \\
\hline 2020-11-05 00:01:00 & 202.560250 & $2020-11-15$ 00:02:00 & 43.208333 \\
\hline$\vdots$ & $\vdots$ & $\vdots$ & $\vdots$ \\
\hline $2020-11-14$ 23:58:00 & 46.803583 & $2020-11-1609: 58: 00$ & 223.961583 \\
\hline 2020-11-14 23:59:00 & 42.562333 & $2020-11-16 \quad 09: 59: 00$ & 215.402417 \\
\hline
\end{tabular}

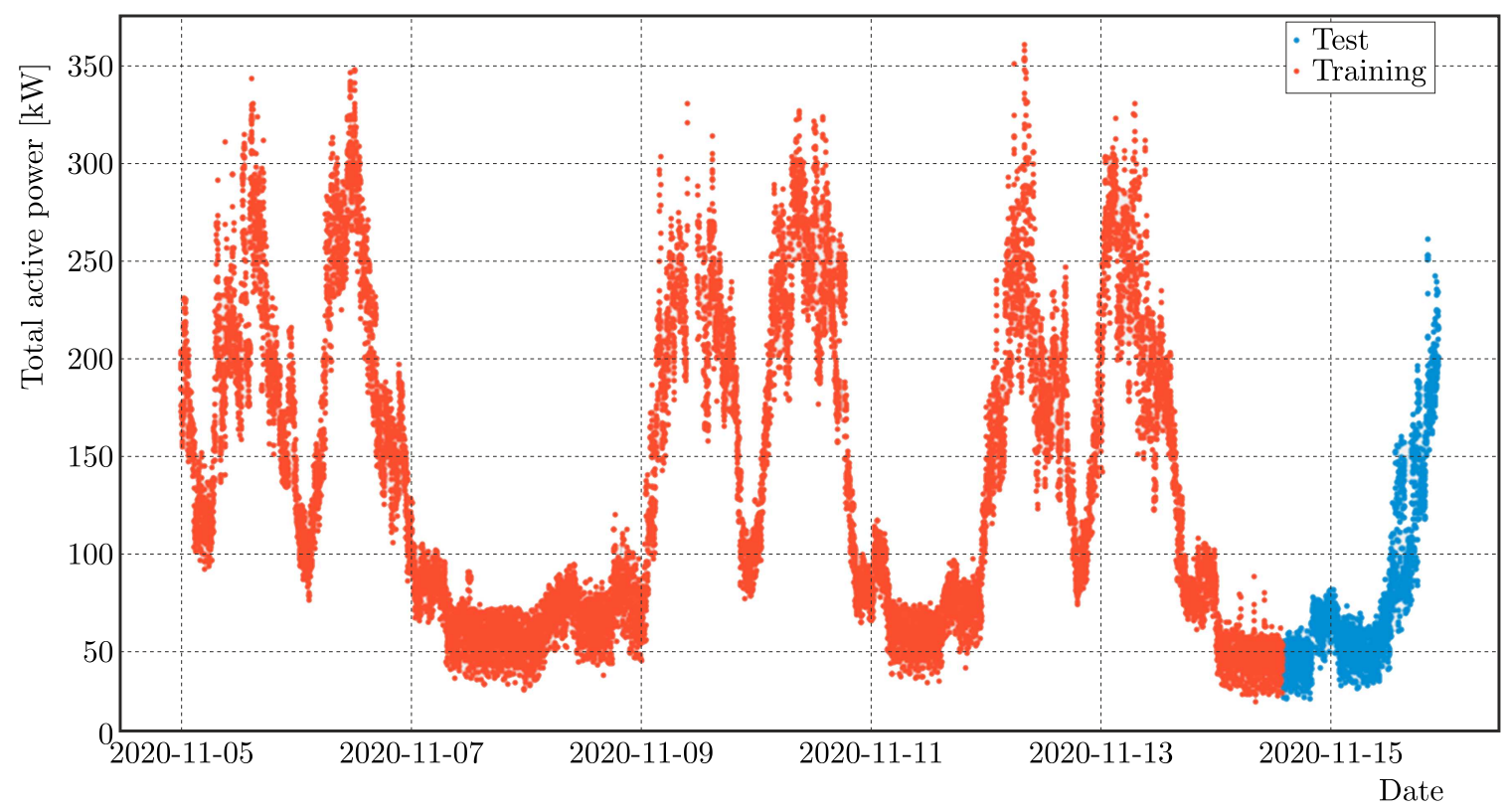

Fig. 6. Division of real data into training and test sets

annual range, which informs that the periodic changes occur cyclically throughout the year. After the algorithm learning process, the model evaluation parameters were determined:

- Time of analysis $40 \mathrm{~s}$,

$-R^{2}=0.86$,

$-\mathrm{MSE}=240 \mathrm{~kW}$,

$-\mathrm{MAE}=14.5 \mathrm{~kW}$.

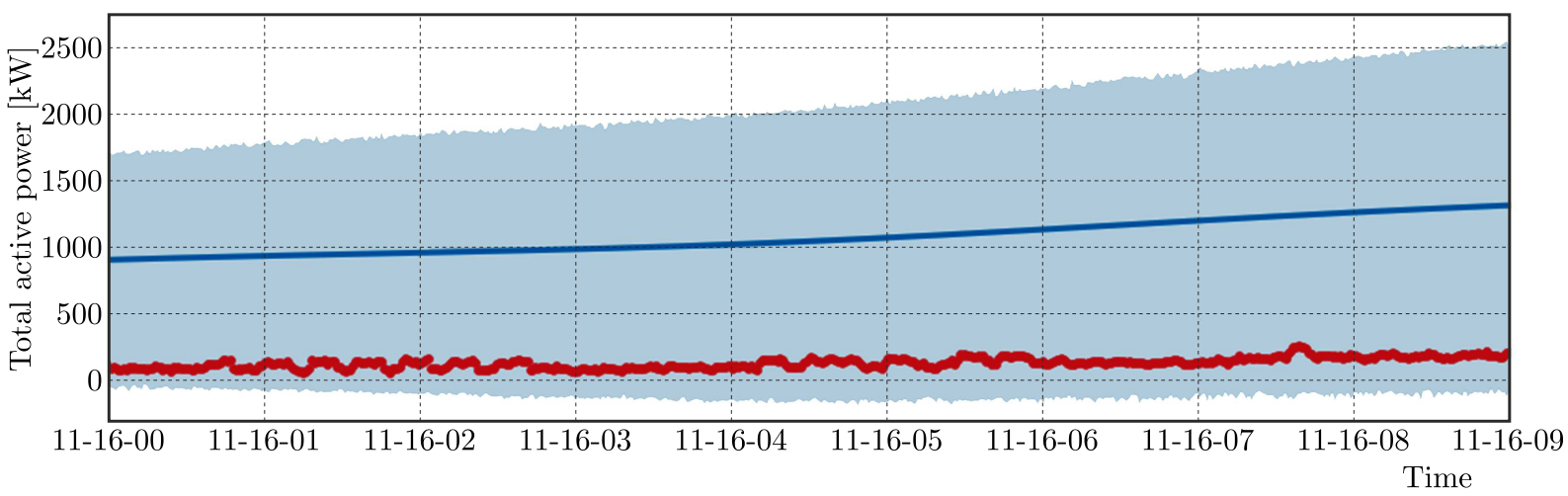

Fig. 7. Value prediction error, blue color error - blue color represents the predicted value, red color the expected value

The model was checked in the prediction of future consumption, which is presented in Fig. 7. 
Unfortunately, the prediction precision and model evaluation parameters are not satisfactory, and it can be assumed that the proposed approach with the Prophet tool incorrectly forecasts the required power value.

\subsection{XGBoost}

The second part of the proposed regression algorithms use data contained in the proposed 4 sets. The analysis started with regression using the Extreme Gradient Boosting (XGBoost) tool, which is effective implementation of gradient increasing for regression and classification problems. XGBoost can also be used for time series forecasting, but the following data need to be transformed into a supervised learning problem (Fig. 5). This method considering both normalized and non-normalized data. Calculated model evaluation parameters for shorter features sets are:

- Without normalizing:

- Time of analysis $180 \mathrm{~s}$,

$-R^{2}=0.857$,

$-\mathrm{MSE}=225 \mathrm{~kW}$,

$-\mathrm{MAE}=9.65 \mathrm{~kW}$.

- After the normalization process:

- Time of analysis $180 \mathrm{~s}$,

$-R^{2}=0.872$,

$-\mathrm{MSE}=201 \mathrm{~kW}$,

$-\mathrm{MAE}=8.86 \mathrm{~kW}$.

Regardless of the historical data whether the values have been normalized or not, the accuracy of the mapping does not exceed $90 \%$, the mean value of the absolute error for both approaches is approx. $9 \mathrm{~kW}$. In comparison with the previously discussed method, the analysis time is doubled. Figure 8 shows the difference between the expected power value and the values determined by the algorithm for the verification data.

In the second step, the regression model was determined for an expanded feature set (with date information). After the learning process, the model evaluation parameters were calculated:

- Without normalizing:

- Time of analysis $180 \mathrm{~s}$,

$-R^{2}=0.872$,

$-\mathrm{MSE}=202 \mathrm{~kW}$,

$-\mathrm{MAE}=9.44 \mathrm{~kW}$.

- After the normalization process:

- Time of analysis $180 \mathrm{~s}$,

$-R^{2}=0.886$,

$-\mathrm{MSE}=180 \mathrm{~kW}$,

- $\mathrm{MAE}=8.81 \mathrm{~kW}$.

XGBoost evaluation parameters allow one to conclude that the increasing of the number of features affects the accuracy of the mapping and reduces the mean error of the active power. However, analyzing Fig. 9, it should be stated that the model incorrectly determines the extreme values (power values greater than the average value), which is unacceptable from the perspective of the analysis of power exceedances in the platform. 


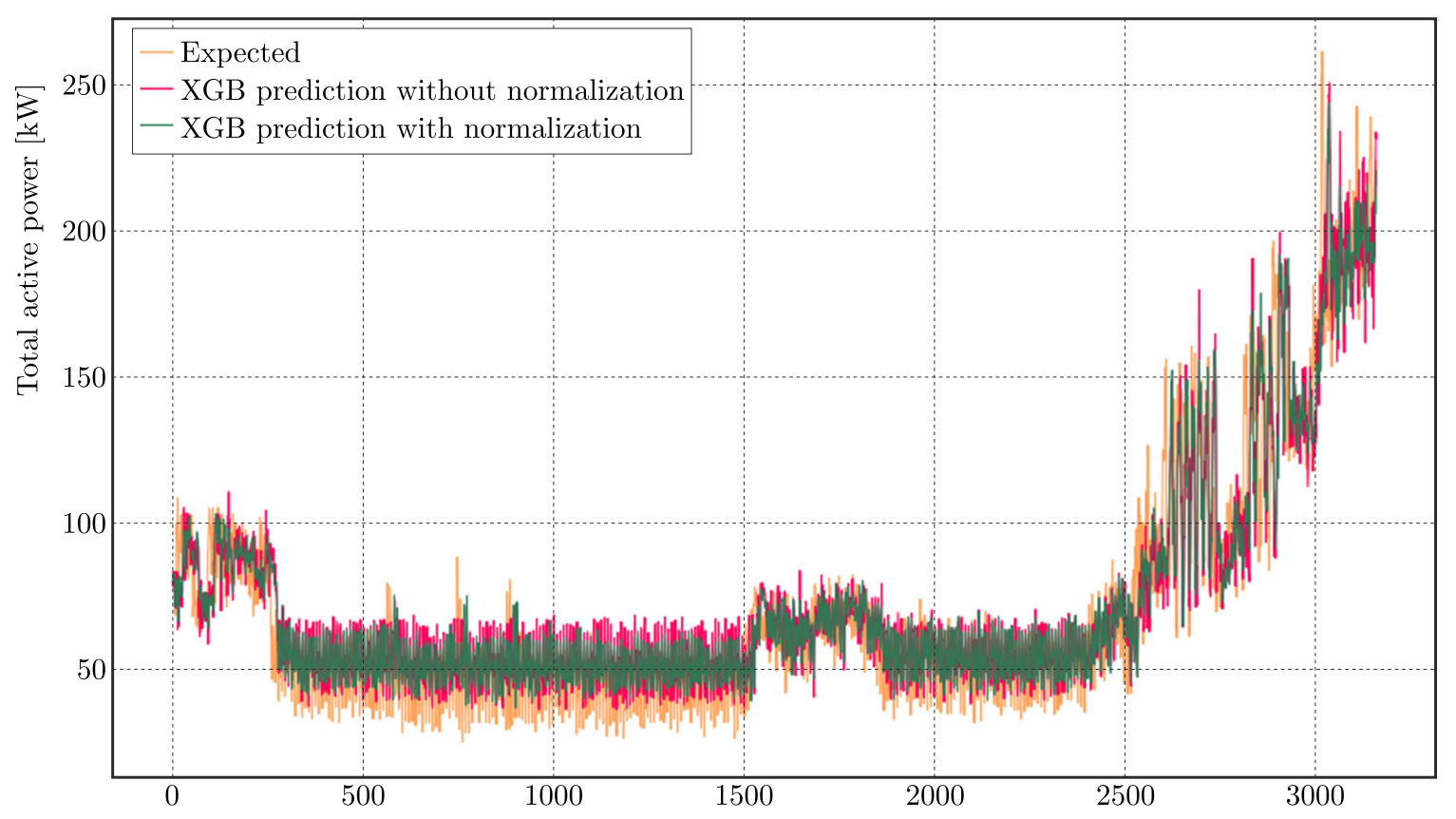

Fig. 8. The difference of the value predicted with the use of XGBoost, green color - the value after the normalization process, red color - the value not taken into account normalization, yellow color - the expected value

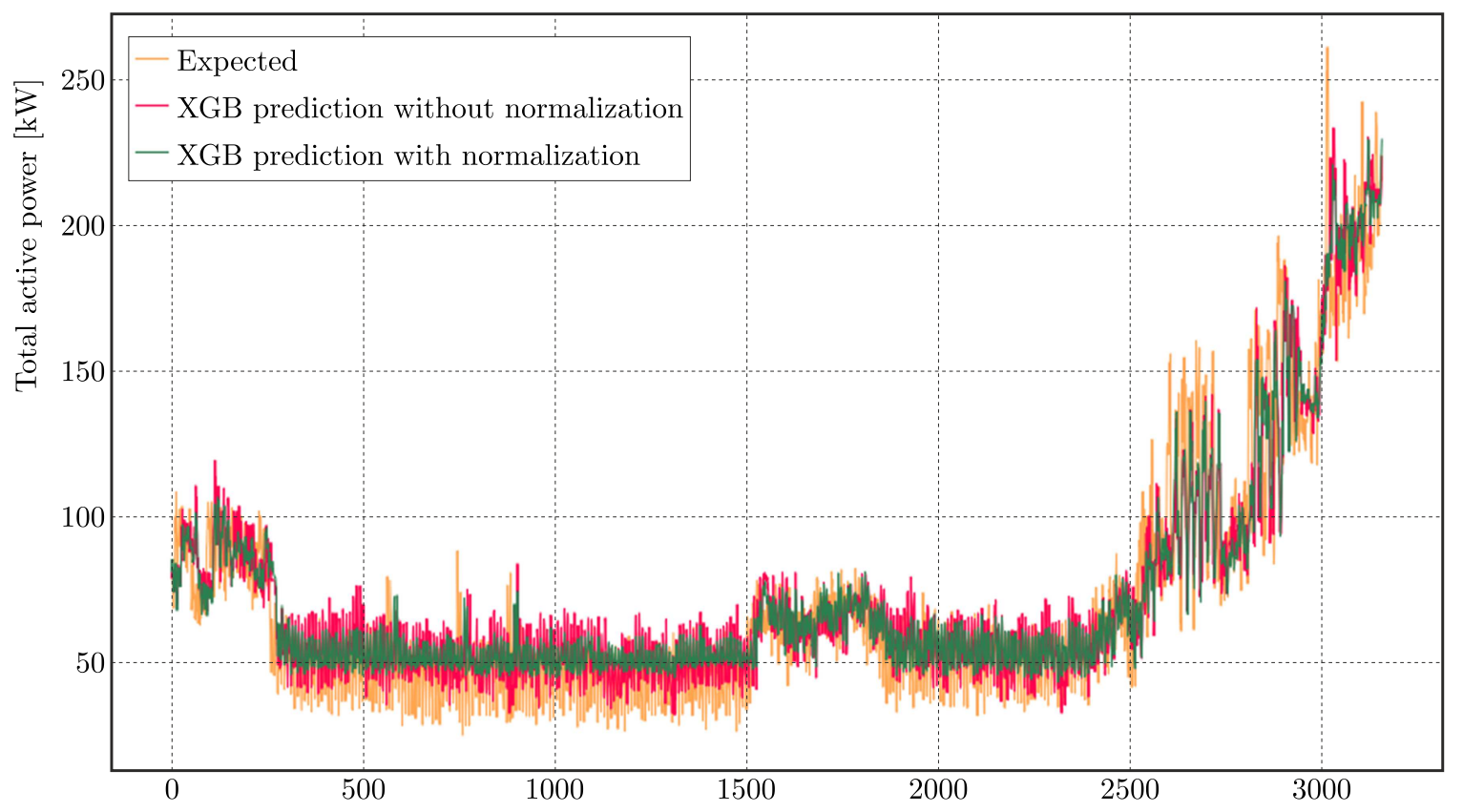

Fig. 9. The difference of the total active power. Prediction with the use of XGBoost - the green color, red color - values without normalization, yellow color represents the expected value

\subsection{Genetic polynomial regression model}

The concept of the regression method assumes determination of a polynomial the arguments of which are the selected features, i.e., historical power values with a time stamp. The task of the genetic algorithm is to select polynomial coefficients in order to maximize the accuracy of power prediction (Temich et al., 2018). The polynomial approximating the space of features to the space of solutions is of the form 


$$
p_{z}=\sum_{n=1}^{N} \sum_{k=1}^{K} a_{z n k} F_{u}^{n k}+b_{z}
$$

where $F_{u}^{n}$ are successive features of the input vector, $a_{z n k}$ and $b_{z}$ are polynomial coefficients determined using the genetic algorithm (AG), $N$ - number input features (in the analyzed case $N=20)$.

On the basis of the research, a second-order polynomial was proposed, i.e., $K=2$. In the evolutionary process, the genetic algorithm was designed to minimize the objective function

$$
F_{c}=1-R^{2}
$$

Due to the properties of the algorithm for searching for the minimum value of the objective function, the proposed function is the inverse of the $R^{2}$ parameter (measure of the function fit), as a result of which minimizing the value of the objective function will result in maximization of the function shape fit. Additionally, the algorithm parameters are:

- Population size $=200$,

- Number of generations $=20,000$.

After the set number of generations was completed, the genetic algorithm completed its operation by determining values of the coefficients $a_{z n k}$ and $b_{z}$ (3.2). Based on the obtained mapping function, the model evaluation parameters were determined:

- Time of analysis approx. a day,

$-R^{2}=0.91$,

$-\mathrm{MSE}=145 \mathrm{~kW}$,

- $\mathrm{MAE}=11,22 \mathrm{~kW}$.

The $R^{2}$ of the method is $91 \%$, which means that the proposed approach is one of the best from the presented. The disadvantage of using the genetic algorithm is the long time for reaching a solution (in the presented case, this time was approximately 24 hours). However, due to the fact that the algorithm performs optimization in the pre-test stage, the learning time is not taken into account. By using AG for prediction, a polynomial is created, which requires basic mathematical operations (multiplication and addition) for the process of determining the predicted value, so this approach is fast and the time of determining the value is negligible.

The disadvantage of the presented solution is the ability to rapidly jump in the value, Fig. 10. A solution to this problem is to increase the degree of the polynomial (e.g., to a 4th or 5th order function). A problem that can also be solved by increasing the order of the polynomial is the time shift of the predicted value.

\subsection{Evolutionary polynomial regression model}

In addition to the genetic algorithm, evolutionary algorithms also include differential evolution (Temich et al., 2019). The construction of the function mapping the feature vector to the expected value of active power is the same as (3.2). The selection of the polynomial coefficients of the mapping function using DE takes place in the population of $P$ individuals, each of which retains an $N K+1$ real numerical value, i.e., each individual contains the number of coefficients equal to the number of vector features $N$ and the polynomial degree $K$. When creating the initial population, the coefficients for $k=1$ get the value obtained from the standard multiple regression algorithm (Lasi et al., 2014). The remaining factors (i.e., $k>1$ ) are determined randomly. The objective function is to minimize the difference between the average value and the current value of the designated functional. The DE algorithm is run for individuals for which 


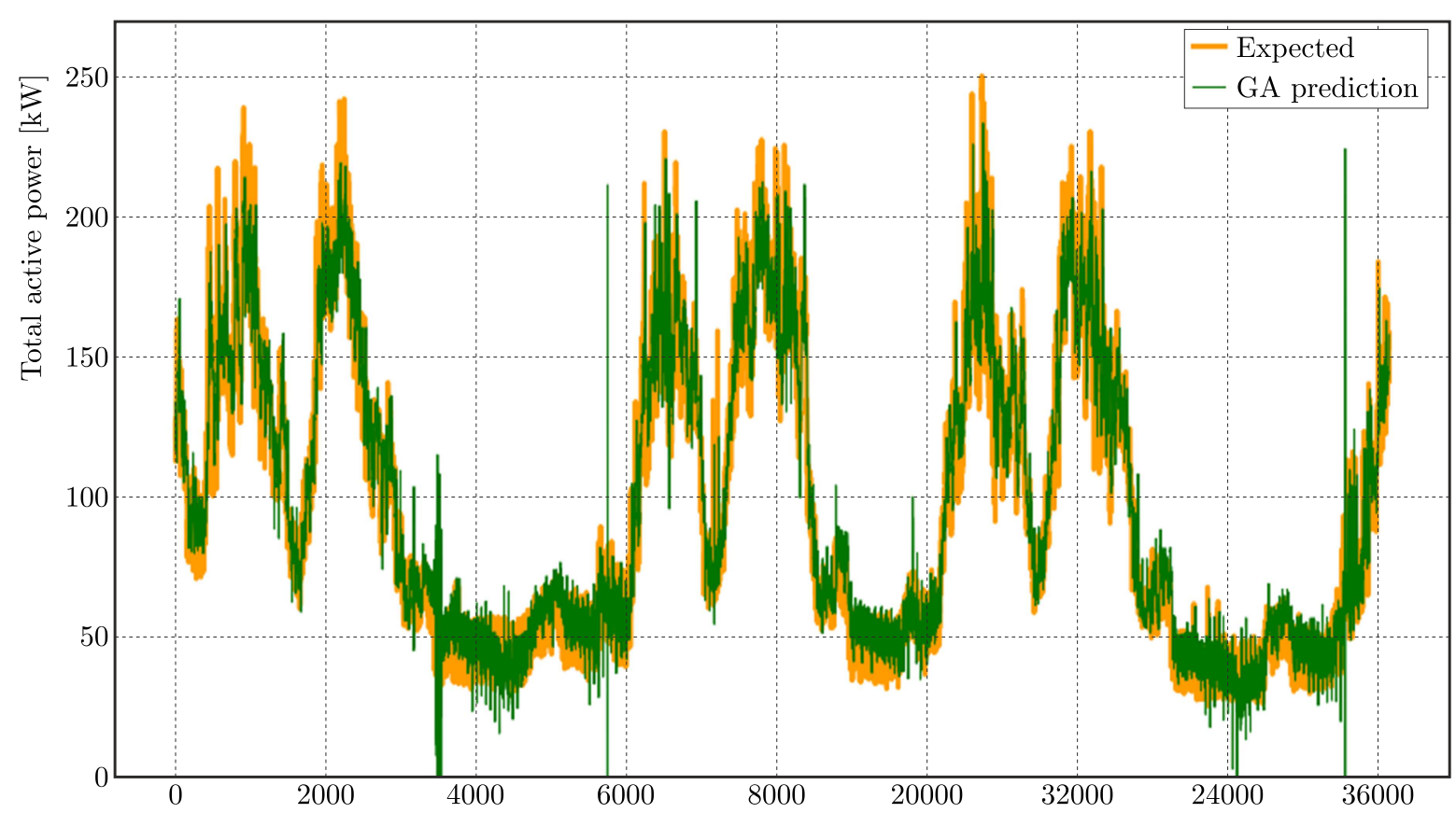

Fig. 10. The difference of the value predicted with the use of GA for data containing time stamps, color green - the value after the normalization process, yellow color - the expected value

each genotype is constructed. To obtain a new individual, the parent genotype $G_{i}$ is crossed with $\widetilde{G}_{i}$, calculated as follows

$$
\widetilde{G}_{i}=G_{i}+w\left(G_{b t}-G_{i}\right)+(1-w)\left(G_{r 1}-G_{r 2}\right)
$$

where $G_{b t}$ is the best genotype found in previous iterations, $G_{r 1}, G_{r 2}$ are randomly determined genotypes. The $w$ parameter is a weight to balance the recombination levels with the specific parts. During the crossing operation, the original genes can be replaced with new ones with a mutation probability of 0.5 .

Based on the research, a third-order polynomial was proposed, i.e., $K=3$. In the evolutionary process, the differential evolution algorithm was supposed to minimize the objective function which was identical to the function introduced for genetic algorithm (3.3). Additionally, the algorithm parameters were introduced:

- Population size $=2000$,

- Number of generations $=2000$.

After the set number of generations was completed, the DE algorithm completed its operation by determining values of the coefficients $a_{z n k}$ and $b_{z}$ (3.2). Based on the obtained mapping function, the model evaluation parameters were determined:

- Time of analysis = approx. a day,

$-R^{2}=0.93$

$-\mathrm{MSE}=140 \mathrm{~kW}$,

$-\mathrm{MAE}=10.52 \mathrm{~kW}$.

The increasing of the degree of the regression polynomial results improves the accuracy of the mapping function by 2 percentage points ( $R^{2}$ is higher). Compared to the genetic regression, both the mean and absolute error values have been reduced. The time of the differential evolution operation was also 24 hours, but as in the GA testing time is not a critical parameter because the learning process is performed at the pre-test stage. 
According to Fig. 11, it should be stated that the algorithm largely adjusts to the expected values but does not reach the peak ones. A solution to this problem is to increase the degree of the polynomial to follow the curve more accurately. In the differential regression, a time shift problem arises which, as suggested for the genetic algorithm, can be solved by increasing the order of the polynomial.

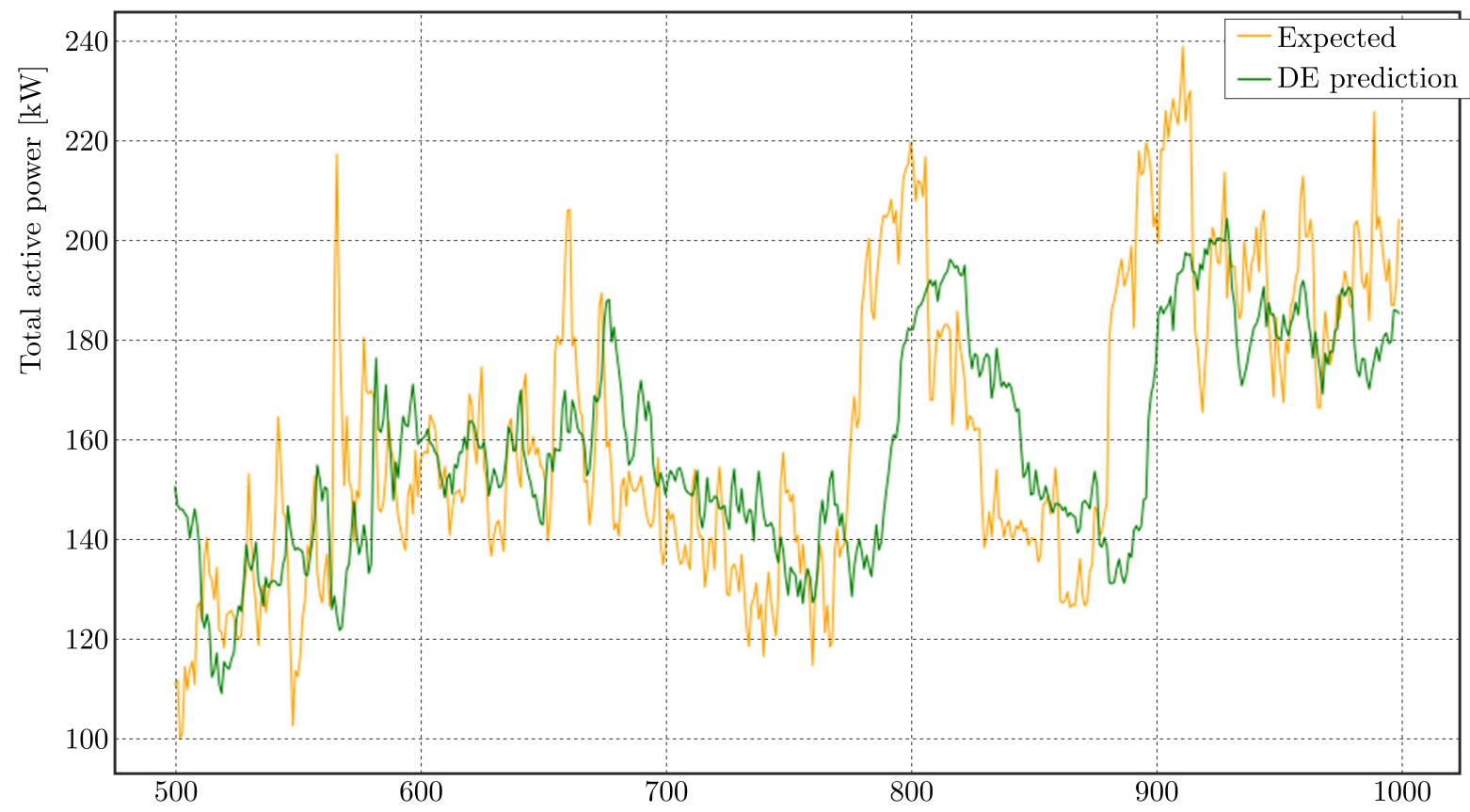

Fig. 11. The difference in value mapping, predicted DE for data including time stamps for the shorter time window

\section{Summary}

This article is aimed at validating various methods of predicting future signal behavior. The analysis was performed in the case of the total active power in a company in order to improve the quality of production management, prevent production outage and machine damage.

The presented solutions use methods from the area of Machine Learning (Park and Son, 2021; Chen et al., 2020; Santra and Lin, 2020), i.e. genetic algorithms, random forests or SARIMA model (Papalexopoulos and Hesterberg, 1990). As a part of the work, 13 prediction methods were proposed, four of them were discussed more precisely. To review the usage of the methods in more detail, in addition to those discussed, the following were also examined: ARIMA/SARIMAX, decision trees (Soutullo et al., 2020), ensemble regression (Gawin, 2017; Aslam et al., 2020), Gaussian regression (Vassalo et al., 2020; Lubbe et al., 2020), artificial neural networks in feed forward structure (Temich et al., 2018) and LSTM structure (Aslam et al., 2020; Skrobek et al., 2020). Every method was compared in accordance with evaluation parameters, which consisted of values of the degree of fit (R2), mean error (MSE) and absolute error (MAE). A collective comparison of the results obtained for each of the methods is presented in Table 3.

Based on the obtained results, it can be stated that the most accurate prediction was obtained for the SARIMAX method, the evolutionary and genetic regression model, and the Gaussian process. The advantage of the SARIMAX method is the greatest degree of curve adjustment to the expected values as well as the time of the algorithm learning process. The use of a genetic or evolutionary regression model offers more flexibility despite the long learning time, another advantage of this two is the ease of implementation in any environment. Despite achieving a high 
Table 3. Summary of the results obtained for each of the introduced methods

\begin{tabular}{|l|c|c|c|}
\hline \multicolumn{1}{|c|}{ Method } & $R^{2}$ & MSE & MAE \\
\hline \hline Prophet & 0.86 & 240 & 14.5 \\
\hline SARIMAX & 0.97 & 178 & 9.8 \\
\hline XGBoost & 0.89 & 180 & 8.8 \\
\hline CatBoost & 0.87 & 203 & 9.5 \\
\hline Fine tree & 0.92 & 224 & 11.2 \\
\hline Ensemble regression & 0.95 & 140 & 8.4 \\
\hline Gaussian proces regression & 0.97 & 100 & 7.5 \\
\hline Genetic model (GA) & 0.91 & 145 & 11.2 \\
\hline Evolutionary model (DE) & 0.93 & 140 & 10.5 \\
\hline Feed Forward Network & 0.85 & 240 & 11.7 \\
\hline LSTM & 0.83 & 266 & 12.6 \\
\hline
\end{tabular}

fit, the Gaussian process is a dedicated method and leada to a risk for dependencies (waveforms) that do not show periodicity (i.e., in other companies), which may cause that the accuracy of the prediction will be far from the expected.

In summary, the performed analysis of the results obtained for the best of the methods presented, the implementation decision should be made between the SARIMAX method and the GA or DE model. However, one should be aware that these solutions are not universal due to the unique nature of each enterprise and require an individual approach to maximize production efficiency.

The structure and universality of the proposed algorithms enable their use on any type of electrical or mechanical signals. The presented article shows possibility of application to energy consumption prediction, whereas the study includes accuracy analysis for the vibration signal, the results of which will be published in the near future. The development of appropriate signal optimization algorithms is required to implement changes in modern plants in order to increase efficiency and introduce savings in production processes. The proposed algorithms, together with human experience, greatly facilitate enterprise management and constitute an integral part of the development of enterprises in the future that fit into the idea of Industry 4.0.

Acknowledgment

This work was supported by the European Union under the ROP WSL for 2014-2020 "Development of the Nazca 4.0 production optimization platform through R\&D".

\section{References}

1. Adenuga O.T., Mrofu K., Ramatsetse B.I., 2020, Exploring energy efficiency prediction method for Industry 4.0: a reconfigurable vibrating screen case study, Procedia Manufacturing, 51, 243-250

2. APAGROUP-1, "IPOE", https://www.apagroup.pl/en/seop (06, 2021)

3. APAGROUP-2, "NAZCA4.0", https://www.apagroup.pl/nazca-4-0 $(06,2021)$

4. Aslam Z., Javaid N., Ahmad A., Ahmed A., Gulfam S.M., 2020, A combined deep learning and ensemble learning methodology to avoid electricity theft in smart grids, Energies, 13, 21

5. Bonilla S.H., Silva H.R.O., Da Silva M.T., Gonçalves R.F., Sacomano J.G., 2018 Industry 4.0 and sustainability implications: A scenario-based analysis of the impacts and challenges, Sustainability, 10, 10 
6. Chen C.-W., Li C.-C., Lin C.-Y., 2020, Combine clustering and machine learning for enhancing the efficiency of energy baseline of chiller system, Energies, 13, 17

7. Díaz Redondo R., Vilas A., Rodríguez A., 2020, Inferring energy consumption patterns in public buildings, Conference Paper, 11, 33-39, https://doi.org/10.1145/3416011.3424753

8. GAWIN Ł., 2017, Heterogeneous ensemble of specialised models - a case study in stock market recommendations, International Symposium on Methodologies for Intelligent Systems, ISMIS 2017, 728-734

9. Gościniak T., Wodarski K., 2019, Effectiveness of using the method of artificial intelligence in maintenance of ICT systems, Management Systems in Production Engineering, 27, 1, 40-45

10. Gruber F.E., 2013, Industry 4.0: a Best Practice Project of the Automotive Industry, Digital Product and Process Development Systems, Berlin, Heidelberg, 36-40

11. Lasi H., Fettke P., Kemper H.G., Feld T., Hoffmann M., 2014, Industry 4.0, Business and Information Systems Engineering, 6, 239-242

12. Leme J.V., Casaca W., Colnago M., Dias M.A., 2020, Towards assessing the electricity demand in Brazil: Data-driven analysis and ensemble learning models, Energies, 13, 6

13. Lubbe F., Maritz J., Harms T., 2020, Evaluating the potential of Gaussian process regression for solar radiation forecasting: A case study, Energies, 13, 20

14. Papalexopoulos A.D., Hesterberg T.C., 1990, A regression-based approach to short-term system load forecasting, IEEE Transactions on Power Systems, 5, 4, 1535-1547

15. PARK H., Son J.-H., 2021, Machine learning techniques for THz imaging and time-domain spectroscopy, Sensors, 21, 4, 1186

16. Podder A.K., Islam S., Kumar N.M., Chand A.A., Rao P.N., Prasad K.A., Logeswaran T., Mamun K.A., 2020, Systematic categorization of optimization strategies for virtual power plants, Energies, 13, 23

17. Pollak A., Hilarowicz A., Walczak M., Gąsiorek D., 2020, A framework of action for implementation of Industry 4.0 an empirically based research, Sustainability, 12, 14

18. Prophet, 2021, Facebook Prophet, http://facebook.github.io/prophet/docs/quick_start.html (07, 2021)

19. Ptasinski W., Pollak A., Temich S., Gasiorek D., 2021, The influence of the condition of bearings on the maintenance of production processes (in Polish), Management and Quality Zarzadzanie i Jakość, 3, 1, 60-73, ISSN 2658-2104

20. SAntra A.S., Lin J.-L., 2019, Integrating long short-term memory and genetic algorithm for short-term load forecasting, Energies, 12, 11

21. Skrobek D., Krzywanski J., Sosnowski M., Kulakowska A., Zylka A., Grabowska K., Ciesielska K., NowaK W., 2020, Prediction of sorption processes using the deep learning methods (long short-term memory), Energies, 13, 24

22. Soutullo S., Giancola E., Jiménez M.J., Ferrer J.A., SÁnchez M.N., 2020, How climate trends impact on the thermal performance of a typical residential building in Madrid, Energies, 13, 237

23. Temich S., Chruszczyk L., GrzechcA D., 2018, Identification of the specification parameters for a voltage controlled oscillator using an artificial neural network with a genetic algorithm, Elektronika ir Elektrotechnika, 24, 6, 42-49

24. Temich S., Golonek T., Grzechca D., 2019, Design an identification function to reduce the computational resources on the testing process of an analog electronic circuit, Elektronika ir Elektrotechnika, 25, 3, 25-33 
25. Tkachenko V., Kuzior A., Kwilinski A., 2019, Introduction of artificial intelligence tools into the training methods of entrepreneurship activities, Journal of Entrepreneurship Education, 22, 6

26. Wang B., Tao F., Fang X., Liu C., Liu Y., Freineit T., 2020, Smart manufacturing and intelligent manufacturing: a comparative review, Engineering,

https://doi.org/10.1016/j.eng.2020.07.017

Manuscript received April 20, 2021; accepted for print June 7, 2021 